\title{
Existe-t-il une politique italienne des télécommunications ?
}

\section{Emmanuel Négrier}

\section{Citer ce document / Cite this document :}

Négrier Emmanuel. Existe-t-il une politique italienne des télécommunications ?. In: Quaderni, n³2, Printemps 1997. Italie, pouvoir et communication. pp. 107-123.

doi : 10.3406/quad.1997.1194

http://www.persee.fr/doc/quad_0987-1381_1997_num_32_1_1194

Document généré le 17/10/2015 


\section{Dossier}

\section{Existe-t-il une politique italienne \\ des télécom- munications?}

Du campanilismo à l'attention toujours manifestée à l'égard des grands clivages entre trois Italies, l'impasse faite en Italie sur l'Etat ou la nation rend problématique l'idée même de politiques publiques italiennes. Celles-ci supposent en effet un lieu central d'articulation des intérêts et de négociation des programmes, qui semble singulièrement déficient ou pour le moins limité, qu'il s'agisse de Rome, de Milan ou de Turin d'ailleurs. A contrario, c'est sans doute ce qui explique la curiosité, que les chercheurs italiens manifestent avec leurs homologues espagnols, pour une approche régionalisée de la représentation des intérêts (Cammelli 1989).

Le secteur des télécommunications n'en confirme que partiellement le bien-fondé. Il a pour originalité, contrairement à la plupart des pays européens (sauf le Danemark), de s'être d'abord développé sur une base

Chercheur CNRS CEPEL-U.R.A. 1267 Montpellier I territorialement fragmentée. Découpé en plusieurs zones géographiques exclusives, l'espace italien est resté durablement nonnational du point de vue des télécommunications. La rationalisation, intervenant dans les années 1950, déboucha sur une répartition, segmentée elle aussi, des fonctions d'opérateur(1). Cependant, si cette fragmentation reste atypique du point de vue topologique, et très difficilement réformable comme on le verra, c'est aussi parce qu'existe bel et bien ce lieu central de régulation des 
intérêts, qui voient interagir les acteurs classiques d'un tel secteur : les industriels, les opérateurs de réseaux, les syndicats et le personnel politique national. Cette distribution classique des acteurs peut ne pas pratiquer les mêmes registres, les premiers et seconds rôles n'étant pas forcément les mêmes qu'ailleurs par exemple, elle n'en demeure pas moins l'indice d'une scène, nationale, de définition et de mise en œuvre des politiques publiques.

Dans ce paysage singulier, on trouve des éléments d'apparence archaïque, sentiment renforcé par la lecture de la chronique redondante des experts du système, en général hostiles au "conservatisme" de ses arrangements. Mais c'est aussi un des pays avancés dans la refonte territoriale des opérateurs publics, sans parler du radicalisme annoncé des stratégies de privatisation, qu'il s'agisse du secteur des télécommunications ou de l'économie publique italienne prise globalement.

Une première lecture de la genèse des télécommunications donnera toute sa place à la notion de fragmentation, par laquelle est souvent appréhendée la singularité italienne. L'analyse de la pénétration en Italie des réformes européennes mettra en lumière une conception particulière des relations entre service public, monopole et systèmes d'intérêt. La troisième thèse, très souvent mise en avant pour expliquer (ou renoncer à le faire) les traits originaux des politiques italiennes, tire argument de la notion de politisation. Nous verrons qu'elle mérite largement d'être nuancée.

\section{LIBÉRALISATION DES TÉLÉCOMMUNICATIONS ET RÉSEAUX D'INTÉRÊTS}

\section{Gouverner la fragmentation}

L'une des dimensions héritées du service public en matière de réseau se trouve notamment dans les transactions, techniques et politiques, auxqucllcs unc organisation consent en échange, le plus souvent, de l'exclusivité d'un service ou d'une infrastructure. Si l'analyse est valable quel que soit le contexte dans lequel les politiques se déploient, il est devenu trivial de noter que cette esprit de transaction s'illustre en Italie jusqu'à la caricature. Loin de n'être qu'une manifestation purement bureaucratique ou politicienne, la segmentation historique de l'organisation publique des télécommunications italiennes procédait au contraire d'une articulation très spécifique des intérêts industriels et gouvernementaux. L'ASST (Agence d'Etat pour le Service Téléphonique, seul organisme à statut administratif dans le système) notamment, a moins dû sa 
survivance à la simple niche de postes et d'influence qu'elle avait constituée pour le syndicalisme démocrate-chrétien (la CISL) qu'à la convergence d'intérêts qu'elle avait su actualiser au sommet avec les principaux industriels. D'une certaine manière, cette organisation constituait la principale garantie pour les industriels (Fatme, Ericsson, Italtel, Siemens, Phillips...) d'une stabilité dans le changement, d'une "lotizzazione" à l'envers en quelque sorte, où les parts de marché de chacun des protagonistes (des câbleries, des équipements de commutation ou des terminaux) se voyaient figées, hors de toute considération de développement technologique ou de compétition frontale cntrc fournisscurs. Cette fragmentation avait d'ailleurs des conséquences sur la stratégie de la Confindustria, organisation patronale italienne, longtemps incapable d'arbitrer entre les revendications générales d'un réaménagement institutionnel et, au delà, d'une efficience accrue de l'offre de télécommunications, et la stabilité exigée par les industriels du secteur, par ailleurs membres de la confédération. Il faudra attendre le mouvement constitué autour de la Commission Morganti, à la fin des années 80 , pour voir apparaître un discours cohérent de l'organisation patronale.

Dans cette organisation sectorielle, le rôle des élites publiques était assez diffférent des modèles connus ailleurs en Europe. Le rôle de pilotage du secteur, notamment par la maîtrise de la recherche-développement, et la constitution de champions nationaux s'est très tardivement incarné, et encore partiellement, dans la constitution d'Italtel. Du reste, c'est plus un rôle d'ajustement des positions respectives, sans prétention de politique industrielle, que jouait l'appareil public. C'est sans aucun doute une des origines, comme une des conditions de perpétuation du rôle important des élites politiques à la tête des entreprises publiques et des sphères administratives. La fonction de médiation du personnel politique, démocrate-chrétien exclusivement au départ, puis graduellement et sélectivement ouvert aux autres formations politiques $\mathrm{du}$ pentapartito, était d'autant plus favorisée qu'il s'appuyait lui-même sur une fragmentation des intérêts et une délégation de l'innovation industrielle.

La structure du syndicalisme des télécommunications apportait une touche supplémentaire au tableau de la fragmentation. A l'image des courants se répartissant dans les différentes niches institutionnelles, les organisations syndicales ont eu une influence très différenciée selon qu'il s'agissait de l'ASST ou des organes chapeautées par l'IRI. Au sein de l'ASST, la représentation du personnel est restée dominée, avec 
un très fort taux de syndicalisation, par l'organisation proche de la Démocratie Chrétienne, la CISL. Celle-ci, historiquement influente dans le Veneto, au Nord-Est de l'Italie, a bénéficié de la consolidation du pouvoir démocrate-chrétien au sein de l'ASST pour y constituer un véritable bastion : environ $90 \%$ des salariés appartenaient à ce syndicat, dont étaient régulièrement issus les dirigeants eux-mêmes, comme M.Zerella par exemple. Progressivement, la maitrise de l'appareil syndical s'est nourrie des avantages obtenus par celui-ci pour ses salariés, en comparaison bien supérieurs à ceux dont bénéficiaient les employés des sociétés nationales de statut privé, au sein de l'IRI.

A l'opposé, la représentation syndicale majoritaire au sein de ces dernières s'est très nettement orientée du côté des organisations proches des partis socialistes et communistes : la CGIL. Celle-ci n'atteint toutefois pas le niveau de domination dont bénéficie la CISL au sein de l'ASST, même si un calcul effectué sur l'ensemble du secteur de l'exploitation publique donnerait à ce dernier syndicat, vu le nombre, une majorité de la représentation salariée.

Du point de vue formel, les conditions d'une dynamique corporatiste au sein du secteur sont donc particulières : elles passent, à l'image du système de partage partisan des influences, par une segmentation idéologique et organisationnelle. De plus, cette division même de l'appareil syndical, qui interdit de penser, comme dans les types idéaux du modèle corporatiste, à une scène centrale de régulation des conflits, empêche également de la qualifier de corporatisme sectoriel, comme on l'a vu identifié pour la France (Muller 1990). A l'intérieur existent trop de divergences d'appartenance pour voir se couler des professions ou même des métiers sous la bannière d'un seul représentant. La capacité d'influence des syndicats est, par ailleurs, sérieusement limitée par les autres sources de légitimité politique interne. On peut, jusqu'à un certain point, défendre l'idée qu'au sein de l'ASST, l'osmose entre syndicat et personnel dirigeant de la DC ait conduit à une production institutionnelle de type micro-corporatiste. Par contre, au sein des autres institutions de l'IRI, les deux groupes sont conduits à des négociations séparées des premières et autrement conflictuelles. Par ailleurs, la force des syndicats, au sein de ces dernières, a tendance à décliner, comme dans les autres secteurs économiques, publics et privés.

Cette structuration des syndicats ne leur interdisait pas cependant de jouer un rôle parfois déterminant dans les conditions de mise en œuvre des politiques publiques. Ils apparaissaient comme des organisations 
segmentées par enclaves au sein de l'appareil public. Cette segmentation, qui pouvait passer pour un des hasards des télécommunications italiennes, était d'une remarquable stabilité, redoublant ainsi la forme de pénétration partisane du secteur. Elle constituait aussi une des plus sûres barrières à la privatisation, compte tenu de l'imbrication extrême des fonctions, modalités financières et intérêts en jeu.

\section{Réformer la segmentation}

Ces trois facteurs (arrangement sectoriel des intérêts industriels, médiation partisane et encadrement syndical fragmenté) expliquent pour l'essentiel le caractère extrêmement laborieux du processus de réforme organisationnel. Devant tenir compte de multiples positions acquises et actualisées par les différents protagonistes, ce processus affrontait ensuite les différentes stratégies des opérateurs publics (SIP, STET, ASST...) pour la concentration autour d'eux-mêmes des différents segments : d'où la succession de projets de super-STET, super SIP... aussi volontaristes qu'inaboutis. Le réseau d'intérêts favorable au statu quo ante était ainsi structurellement plus puissant que celui qui dénonçait l'inefficience du système et son inadaptation aux nouvelles normes d'action définies à l'échelle européenne. Qu'il s'agisse des experts indépendants, comme Franco
Vergnano ou Franco Morganti, de ceux dépendant de la Confindustria, d'une partie de l'élite de la SIP ou de la STET, et enfin de l'association des (gros) utilisateurs, qui pourraient caractériser le réseau de soutien à une réorganisation rapide, force est de constater leur durable difficulté à pénétrer le cœur du système d'action, c'est-à-dire à s'imposer comme un réseau intellectuellement et stratégiquement dominant.

De sorte que c'est en fonction d'un changement plus global, où la disqualification profonde de la Démocratie Chrétienne et du système C.A.F. (Craxi-Andreotti-Forlani) le disputait à la dramatique situation des finances publiques, que ce réseau cst parvenu d'une part à atténuer ses propres contradictions (Super-STET / Super-SIP) et d'autre part à incarner pour le secteur la nouvelle frontière politique d'action, le "mani pulite" des télécommunications en somme.

Le processus de réorganisation institutionnelle, qui a concrètement débouché sur le passage au sein de l'IRI, de l'ASST, rebaptisée IRITEL, puis l'intégration de l'ensemble des opérateurs, dont IRITEL, au sein du groupe Telecom Italia, s'est fait selon un calendrier globalement respecté, à la suite de longues négociations entre syndicats, groupes d'intérêts industriels, professionnels et opérateurs publics. 
Le "riassetto" n'est cependant pas parvenu à régler définitivement les questions de frontières internes au groupe, comme en témoignent les débats actuels qui concernent le statut de la radiotéléphonie mobile. Après la décision de l'"Antitrust" italien d'interdire pour l'instant à la SIP de lancer son propre service de radiotéléphone mobile GSM, pour permettre une entrée concurrentielle dans de secteur, la question reste posée du mode institutionnel de gestion des télécommunications mobiles à l'intérieur de Telecom Italia. Le débat tourne autour de trois solutions, qui sont autant de marque de la permanence des clivages historiques au sein de l'appareil public : la gestion confiée à la SIP directement; l'autonomisation de celleci au sein de la STET; la privatisation pure et simple. De ce point de vue, les changements gouvernementaux récents auront un rôle fondamental d'arbitrage. Par ailleurs, les changements sectoriels et politiques ont produit leurs propres effets sur le plan syndical. Avec la disqualification brutale des accords implicites entre la démocratie chrétienne et "son" syndicalisme dans la fonction publique, se trouve posé le problème de la représentation des intérêts sociaux au sein du nouveau groupe. De nouvelles formes de syndicalisme (l'introduction d'un syndicalisme "autonome" proche du mouvement "Forza Italia", la montée en puissance d'un syndicalisme leghiste à travers le Sindacato Autonome Lombardo) émergent parallèlement à une homogénéisation tentée, autour de la CGIL (gauche) notamment, au sein du groupe. Cette tendance au changement est d'autant plus forte que de nouvelles règles de représentation syndicale ont été récemment instituées : elle procède désormais d'une élection des représentants par entreprise et non plus d'un simple décalque des affiliations par adhésion.

Les tendances au changement dans le système national de télécommunication italien mettent en évidence le poids relatif des évolutions technologiques et réglementaires internationales pour expliquer les mutations dans l'existence et la consistance du service public. Ceci ne signifie pas que cet ensemble de mouvements n'ait aucun impact sur les stratégies d'acteurs et les politiques publiques. Ils contribuent au contraire à nourrir les représentations et controverses à l'intérieur du système d'action collective, même s'il n'est pas le moteur principal de ses recompositions politiques, sur les points suivants :

- si la pression de la compétition internationale sur les marchés considérés comme les plus rentables fragilise la position du concessionnaire de service public, elle permet aussi à l'opérateur national de faire passer, à l'intérieur du gouvernement, le sentiment de 
l'urgence des réformes, dont l'un des traits est de le rendre plus autonome de celui-ci dans ses stratégies d'entreprise;

- si le développement des innovations technologiques de réseaux et de service, dans un marché d'usages internationalisé, révèle les retards dramatiques accumulés par l'industrie et l'exploitation italienne, il est aussi l'occasion d'un contournement des fragmentations institutionnelles par projet, au bénéfice de l'exploitant national dominant;

- si l'émergence de la réglementation communautaire met en question les compromis passés, entre l'exploitation et l'industrie, sur la base du monopole public, elle renforce aussi la position de la SIP à l'intérieur du système et dans ses relations avec l'Etat dans les politiques de développement territorialisées;

\section{LA PÉNÉTRATION DES NORMES EUROPÉENNES DANS LE SECTEUR TTALIEN DES TÉLÉCOMMUNICATIONS}

La diffusion d'un modèle européen dans le secteur des télécommunications italiennes n'obéit pas à une logique linéaire non plus. Si l'on s'accorde en effet pour reconnaître la dimension plurielle des registres de justification de cette politique, son caractère essentiellement flou, voire parfois contradic- toire, sa diffusion elle-même fait l'objet d'usages également pluriels et de débats de forme et de fond. La question de l'introduction de la concurrence dans les services de télécommunication nous servira de fil conducteur pour en apporter la démonstration.

A travers cet exemple, on verra de quelle manière de nouveaux acteurs utilisent la référence communautaire pour entrer dans le "jeu contractuel" global du secteur, et comment cette référence demeure, alors même qu'elle est assise sur une réelle contractualisation en amont, l'objet de négociations et renégociations continuelles.

Concernant la libćralisation des scrvices de télécommunication, l'Italie fait partie des Etats-membres (parmi lesquels la France et l'Espagne également) qui ont résisté le plus fortement à l'imposition par la Commission européenne d'une politique libérale étendue (Brenac 1994). Aussi bien, le Livre vert de 1987 apparaît-il comme un compromis acceptable, au plan des principes, devant être suivi des dispositions réglementaires sous la forme de directives, édictées au début des années 1990. Si les principes ont été rapidement reçus en Italie comme la nouvelle frontière de l'organisation du secteur, il n'en a pas été de même pour les conséquences pratiques à tirer de ses concrétisations réglementaires. Ainsi la directive 90-388, pu- 
bliée le 28 juin 1990, qui concerne l'introduction de la concurrence dans les services, prévoyait-elle l'abolition, par les Etatsmembres, de tout droit exclusif ou spécial concernant la fourniture de services de télécommunications autres que ceux de téléphonie locale, et l'adoption d'un système d'autorisation non discriminatoire entre une pluralité d'opérateurs. N'ayant pas à subir la pression de l'opérateur public pour son entrée en vigueur italienne, et davantage polarisé sur la refonte institutionnelle du secteur, les gouvernements Amato puis Ciampi ont tenté d'en amoindrir les effets d'ouverture à la concurrence avant de renoncer à intégrer cette directive au droit national. C'est donc par la voie des tribunaux et de la Commission elle-même que le monopole des télécommunications publique a dû être amoindri et précisé. Un tel processus n'est pas exceptionnel en Italie, où la "législation des juges" a, bien avant la dynamique de Mani Pulite, été au principe d'importante évolutions sectorielles (2).

Si cette carence de la réception italienne des normes européennes doit aux circonstances politiques générales (chutes des gouvernements en question et forclusion des délais d'adoption des délégations législatives par le Parlement), elle s'explique aussi par l'extraordinaire complexité du jeu des intérêts organisés (Furlong 1994), qui pro- duit ses propres stratégies de contournement. Quelques exemples le montrent :

1. Dans l'affaire de la directive, l'un des acteurs de premier plan s'est avéré être l'ANUIT, la plus importante association des grands utilisateurs des réseaux de télécommunication. Regroupant essentiellement de grandes entreprises, elle n'avait jusqu'à présent eu qu'un rôle mineur, à cause notamment de ses difficultés à exprimer un intérêt collectif et spécifique dans les différents forums des politiques de télécommunication. La manipulation de la référence européenne constitue pour elle un moyen d'entrer dans le jeu politique, par la bande certes, mais sur un terrain que la Confindustria (la confédération du patronat italien) n'occupe pas réellement (3).

2. Le recours direct à la Commission et aux tribunaux met également en évidence un autre acteur clef : I'"Antitrust" italienne, présidée par l'ancien Président du Conseil italien Giuliano Amato. Son intervention dans le débat sur les télécommunications, et sur la définition d'un régime libéral en ce domaine, est là aussi le résultat d'une lacune et signale un problème de fond dans les politiques publiques italiennes : la question de la régulation du système. Alors que les Etats-membres ont, à travers le Livre vert 
et ses suites, opté pour une séparation rigoureuse entre les fonctions de réglementation et les activités opératrices (4), la laborieuse institutionnalisation d'une Autorité spécialisée traduit les conflits que génèrent en aval les contrats passés en amont. Deux "scénarios" s'opposent clairement :

- celui d'une autorité spécifique au secteur des télécommunications (scénario "continental"), qui serait en quelque sorte l'"objectivation" des relations entre partenaires classique de la régulation sectorielle : le Ministère, les opérateurs, la Commission ; il promeut avant tout la spécificité du secteur des télécommunications, et la nécessité de préserver sa cohérence interne;

- celui d'une fonction de régulation spécifique à l'intérieur de l'actuelle Autorité Antitrust (Amato), aux compétences larges; il promeut d'abord l'efficience réputée de ladite Autorité, dont la naissance a été le théâtre de multiples contraintes (RegoniniGiuliani 1994), depuis la loi lui donnant officiellement corps (1950) jusqu'au début de son fonctionnement réel (1990). Il parie ensuite sur sa capacité à assurer les adaptations réglementaires et contractuelles entre les normes européennes et la réalité italienne.

Le premier scénario est globalement sou- tenu par le centre-droit, l'élite de Telecom Italia, et une partie des entreprises industrielles du secteur. La dépendance de cette autorité spécifique vis à vis du réseau traditionnel des politiques publiques serait à même d'assurer une protection maximale (pour une durée d'adaptation supérieure) des influences dominantes, tout en en négociant les inflexions avec la Commission européenne (en préférant s'appuyer sur la DG XIII, selon la même logique, que sur la DG IV, en charge des questions de concurrence).

Le second scénario est au contraire promu par une large partie des experts (des "professori" notamment, tcls lcs ćconomistes Morganti et Cavazzuti, ou encore R.Prodi, qui structurent une grande partie des positions favorables à une réglementation alternative), du centre-gauche et de la Confindustria. Il parie au contraire sur l'intensité des contacts entre l'Anti-trust actuelle et la Commission européenne, seule en mesure, selon ses partisans, de promouvoir une réglementation libérale du secteur. Il s'appuie de préférence sur la rhétorique de la DG IV, plutôt que sur celle de la DG XIII, et donc sur une théorie aussi limitée que possible de la spécificité du secteur des télécommunications.

Parce que les lignes de fracture entre ces 
positions n'ont pas de traduction politique claire, mais aussi parce que la domination de la "scuola"(5) à la tête des entreprises publiques du secteur n'a pas été discutée à travers la série de réformes institutionnelles passées, la question de l'autorité régulatrice sera longtemps demeurée sans réponse. L'intermédiation de l'Anti-trust elle-même apparaît, faute de mieux, comme un mode transitoire, et volontairement sans cadre clair, de mise en œuvre nationale des contrats communautaires. Pour schématiser sur un ton quelque peu ironique, certains n'hésitent pas à affirmer qu'il existe bel et bien une instance de régulation italienne des télécommunications...mais qu'elle est à Bruxelles! (Morganti 1994)

3. La mise en œuvre des politiques communautaires offre enfin de multiples combinaisons de registres, plutôt qu'un seul. La démonstration, faite dans d'autres domaines, comme celui des politiques structurelles (Smith 1995), vaut pour les télécommunications. La mise sur agenda d'une politique dite d'"Espace Commun d'Information" permet en elle-même de vérifier la pluralité de références et de finalités proposées dans les "méga-contrats" européens. Entre la libéralisation affirmée, et dotée d'un échéancier à géométrie variable, l'attention nouvelle portée sur les risques d'exclusion engendrés par les innovations réglementaires et tech- nologiques (le multimédia et les autoroutes de l'information en général), les impératifs de politique industrielle, l'accent mis enfin sur les effets en termes d'emploi et d'équilibres territoriaux, les contradictions affleurent, et sont parfois le théâtre de conflits : internes à la Commission entre DG IV et DG XIII notamment, entre Etats-membres également, comme on a pu le voir à propos des accords entre France Télécom et Deutsche Telekom (6). Une démonstration du même type pourrait être faite pour l'Italie en ce qui concerne la politique de réduction des disparités territoriales internes à l'Europe et à l'Italie (le Mezzogiorno). Le discours modernisateur, affiché dans la ligne même de la construction européenne (le Piano Europa 1989-1993 par exemple) s'accompagne en effet de la mise en avant d'une exception italienne (de même pour l'Espagne, la Grèce et le Portugal en particulier), elle-même validée par la Commission européenne dans ses propres politiques : les initiatives STAR et TELEMATIQUE qui, bien que réduites dans leur impact financier, permettent bel et bien de marquer un territoire sous le sceau du retard. Ce retard présente de multiples avantages et notamment, pour ce qui nous intéresse, celui d'inscrire dans la contractualisation communautaire la légitimité d'une adaptation différée, d'une contrainte particulière, de la nécessité de maintenir des droits exclusifs, et la situation de 
l'opérateur qui les détient.

\section{QUELLE POLITISATION DES TÉLÉCOMMUNICATIONS ?}

Lorsque le juge Green s'apprêtait à déclencher le démantèlement d'ATT aux États-Unis, il se lança dans une série de consultations afin d'observer la manière dont les différents pays développés géraient leur secteur des télécommunications. A ce titre, il rencontra Carlo Cerutti, administrateur délégué et viceprésident de la STET, à qui il confia sa perplexité devant l'incroyable complexité du système italien. Afin d'expliquer la manière dont on pouvait trouver ses repères dans l'écheveau des structures et officines, ce dernier n'eut qu'une simple réponse : "siamo tutti amici!".

Cette petite anecdote, typiquement italienne, donne un éclairage très particulier sur les conditions de régulation d'un système si fragmenté. Elle intéresse cependant tout chercheur qui souhaite spécifier une champ d'activité sociale avec d'autres moyens que les seuls arguments stratégiques et synchroniques. Dans le cas de l'Italie, le schème de la politisation est souvent considéré comme central. Il recouvre une partie de la réalité analysée ici dans un premier temps : la fragmentation des intérêts organisés irait de pair avec la pénétration des clivages po- litiques et partisans en leur sein. Ce serait même une des caractéristiques plus générales des politiques en Europe du Sud (Schmitter 1995). Nous voudrions discuter ici, dans le cas particulier des télécommunications, le bien-fondé de cette hypothèse d'une "politisation généralisée de l'activité sociale".

Elle recouvre d'abord un certain nombre de réalités empiriquement observables. Le poids historique de la Démocratie Chrétienne à la tête de l'ensemble des institutions du secteur est un premier fait, incontestable. La progressive admission, dans les années 70 et 80 , de certains représentants des partis laïc et socialiste (au moment du pentapartito), renforce l'enrôlement des télécommunications dans la logique de lottizzazione : les administrateurs délégués de ces partis permettent d'élargir la base d'un profond consensus sur l'organisation globale du secteur. Au même titre que les banques, la RAI, les industries mécaniques ou l'administration, les télécommunications fournissent un cadre idéal à cette analyse en termes de politisation. L'observation des carrières à la tête de l'IRI montre que cette politisation remonte loin, à l'origine d'un secteur conçu par les fascistes comme parapublic : contrôlé par le Parti National Fasciste au départ, l'IRI sera ensuite dominé par les partis républicains, de telle sorte que le 
champ politique est devenu central puisqu'il est le lieu majeur de la distribution des emplois et de sélection-reproduction des dirigeants de l'économie (Dormagen 1996).

La pénétration des clivages partisans à l'intérieur du système syndical des télécommunications tend à donner une touche finale à un processus qui paraît dès lors incontournable. Pourtant, cette thèse nous paraît contradictoire avec certains aspects essentiels de la conduite des politiques italiennes de télécommunication :

- elle est d'abord contradictoire avec le fait que la succession de micro-alternance au scin de la Démocratie-chrétienne et du gouvernement n'ait jamais réellement constitué un élément de changement du système. La remarquable permanence des mêmes hommes à la tête des cinq sociétés, puis de Telecom-Italia et de la STET montre un décalage certain entre système politique et élite sectorielle.

- elle est assez contradictoire avec l'absence quasi généralisée d'expertise partisane dans ce domaine. Ce qui est vrai pour la Démocratie-Chrétienne l'est encore plus pour les autres partis. C'est ce qui explique le rôle, très important, qu'ont joué les experts (professeurs, journalistes spécialisés, consultants) dans la construction intellectuelle des problèmes et l'invention de recettes nouvelles d'action publique.

De sorte que le rôle joué, concrètement, par les partis dans l'orientation politique du secteur nous paraît secondaire vis à vis de la fonction essentielle de conduite qu'exerce une élite resserrée, d'une "scuola" dont sont issus tous les dirigeants réels des télécommunications italiennes depuis plus de deux décennies. Infra-partisane dans sa formation, ce groupe s'est appuyé sur une pluralité de soutiens : internes à la DC, voire de l'Opus Dei, du Vatican; au sein du système économique privé, de l'Etat, de certaines partie du monde syndical... Entre cette pluralité de partenaires, un système d'échange politique sur le long terme permettait de sécuriser les arrangements : les marchés pour les industriels; la représentativité et les conquêtes sociales pour les syndicats; les recettes budgétaires pour l'Etat; des postes de prestige pour les partis; et la reproduction de la légitimité des dirigeants pour la scuola. Celle-ci bénéficiait en outre d'une forte capacité de cooptation due au fait que, au contraire de la situation française par exemple (Giraud 1987), il n'existe par de lieu de formation unique, centrale et prestigieuse pour cette élite.

De la sorte, c'est cette élite resserrée qui a conduit, certains diront : pour elle-même, la 
régulation du système ("siamo tutti amici") et notamment l'adaptation idéologique aux nouveaux contextes technologiques, économiques et politiques.

On peut soutenir la thèse de la politisation sur le plan formel (occupation de postes, pénétration partisane dans les institutions publiques et sociétés du secteur...), sur le plan des processus (logiques d'échange politique entre une pluralité d'acteurs intéressés et légitimes). En revanche, il est plus difficile de la repérer sur un plan plus substantiel. La "politisation" des politiques reste un angle mort de l'analyse. La meilleure preuve de cette absence de politisation substantielle, de dessein sectoriel comparable à l'économie du grand projet typique du néo-colbertisme français (Cohen 1992), sera sans doute apportée par les événements en cours après la victoire du pôle de l'Ulivo en avril 1996 : le renversement anticipé par l'élite de la STET et de son dirigeant principal, Ernesto Pascale, des stratégies suivies jusqu'à présent(7) et la convergence totale affichée désormais par ce dernier avec les thèses libérales pronées par l'Antitrust italienne, dirigée par Giuliano Amato. Cette conversion s'illustre en pratique à l'échelle internationale, où la STET multiplie initiatives et alliances, avant même la privatisation annoncée pour le printemps 1997, notamment en Europe. La prise de participation, en octobre 1996, de la STET dans Bouygues Telecom va dans ce sens (8). Néanmoins, cette nouvelle tendance n'empêche nullement la STET de poursuivre, en Italie, sa stratégie d'occupation du maximum de niches de marché. Un exemple de cette coursepoursuite avec les échéances de libéralisation est actuellement fourni par les controverses (entre l'Anti-Trust et la STET) à propos de la mise en place de réseaux de téléphonie urbaine, du même type que le Bibop français (9). Pour la première, il s'agit d'un secteur d'emblée concurrentiel, où Telecom Italia ne peut intervenir sans fausser le jeu de la concurrence. Pour la seconde, il ne s'agit ici que d'une extension physique du réseau fixe, encore soumis au monopolc dc Telecom Italia. En filigrane, il s'agit bien sûr pour cette dernière de prévenir et de retarder éventuellement le choc de la dérégulation, en adoptant une pure logique de marché, même si elle se base sur un monopole désormais crépusculaire.

Une telle deconnexion entre ces trois formes de politisation (formelle, processuelle, substantielle) est liée à la nature particulière de la délégation politique à ce groupe dirigeant. On peut formuler l'hypothèse que le secteur des télécommunications représente, pour la classe politique proprement dite, un investissement coûteux pour une rentabilité aléatoire. Que cette classe ait donc mas- 
sivement déserté l'expertise dans un secteur qui, malgré certaines crises d'ajustement, fournit comme ses homologues européennes un revenu régulier à l'Etat. Qu'enfin les partis politiques aient, en échange de quelques rentes symboliques et matérielles, délégué la régulation du système à un groupe particulier, et par là-même renoncé à conduire réellement ces politiques.

\section{Conclusion :}

Parvenu au terme de notre analyse, on peut ainsi mettre en relation la pluralité de facteurs qui conduisent au paysage si particulier des télécommunications en Italie. La fragmentation, réformée par le riassetto, était fondée sur une pluralité d'institutions mais aussi sur une économie des échanges (avec le secteur privé et les forces syndicales notamment) qui en assurait la stabilité. Cet échange est traversé de conflits et de consensus, où se jouent la légitimité et l'identité des partenaires en présence (Pizzorno 1993). En ce sens, on peut parler d'échange politique. Le rôle des partis politiques dans cette dynamique d'échange ne doit pas être surévalué. S'il joue sa partition dans la lottizzazione, dans la manière dont la domination démo-chrétienne y a durablement imprimé sa marque, il n'a pas significativement produit de corpus de référence stable, $d$ "'idéologie italienne" des télécommunications. La variation dans les orientations stratégiques, les conflits dans l'intensité et les formes de libéralisation, les profonds clivages quant à la pénétration des normes européennes en sont un premier indice. L'appel itératif à des recettes puisées dans d'autres contextes en est un autre, avec le rôle que joue la référence fréquente à l'organisation anglaise du territoire et du management des télécommunications (Négrier et al. 1993). Pour paradoxale qu'elle soit au regard de la thèse de l'éternelle politisation à l'italienne, l'analyse de la situation ouverte avec le nouveau gouvernement Prodi pourrait être celle du retour de la politique dans les télécommunications. 


\section{R É F É R E C E S}

Antonelli C. : New information technology and industrial change : the italian case, Kluwer Academic Publishers, Dordrecht, 1988.

Bianchi A., Scarpitti L. : La politica delle telecomunicazioni in Italia, NomismaLaboratorio di politica industriale, Bologne, 1988.

Bottiglieri B. : SIP. Impresa, tecnologia e Stato nelle telecomunicazioni italiane, Collana CIRIEC di storie d'impresa, FrancoAngeli, Milano, 1990.

Brenac E. : "Néo-libéralisme et politiques industrielles : les conditions et formes différenciées d'un changement de paradigme. L'exemple des télécommunications en Europe" in B. Jobert-B. Théret : Le tournant néo-libéral en Europe, L'Harmattan, Paris, 1994.

Cammelli M. (a cura di) : Regioni et rappresentenza degli interessi, Stato e Mercato, Bologne, 1989.

Cohen E. : Le colbertisme "hightech". Economie des Télécoms et du grand projet, Editions Hachette Pluriel, Paris, 1992.

Dormagen J.Y. : "Profession politique et politisation des professions en Italie. Le cas des conseillers d'administration de l'IRI (1939-1990)", Communication au Congrès de l'AFSP, Aix-en-Provence, avril 1996, 23 p. P.Furlong : Modern Italy. Representation and reform, Routledge, London, 1994.

Giacalone D., Vergnano F. : "La guerra del telefono. Le telecomunicazioni, la gestione, la politica" Il Sole 24 Ore Libri, Milan, 1990. Giacalone D., Vergnano F. (dir.) : "Tamburi di latta. L'Italia dei telefoni nella sfida mondiale" Il Sole 24 Ore Libri, Milan, 1991.

Ginsborg P. : Storia d'Italia dal dopoguerra a oggi. Sociétà e politicà 1943-1988, Einaudi, Turin, 1989.

C.Giraud : Bureaucratie et changement, L'Harmattan, Paris, 1987.

Morganti F. : "Le telecomunicazioni", Progetto Infrastrutture SIPI Ed. Rome, 1990. F.Morganti : "I Vincoli imposti dalla normativita italiana" in Le autostrade dell'informazione : opportunità di business nelle telecomunicazioni, Il Sole 24 ore Convegni, Milan 1994, dactylo 11 p. + annexes.).

Muller P. : Les politiques publiques, PUF, Que sais-je ?, Paris, 1990.

Musso P. : "La longue marche à l'organisation`des télécommunications italiennes" in La Lettre du SPES, $\mathrm{n}^{\circ} 13$ spécial-Italie, $\mathrm{Pa}$ ris, 1988.

Musso P., Pineau G. : L'Italie et sa télévision, Champs Vallon Editeur, Paris, 1991.

Musso P., Richeri G. : La convergence de l'audiovisuel et des télécommunications en Italie, Rapport CNET-MaknoMedia, MilanParis, 1989.

E.Négrier et al. : Les politiques publiques 
de télécommunications en Europe du Sud. Service public et dynamiques territoriales des intérêts, CEPEL-Commissariat Général du Plan, Montpellier, 1993 (avec A.Briole, L.De La Torre, R.Lauraire).

E. Négrier : Identité européenne et politiques publiques: le cas des télécommunications, Rapport pour le Programme de recherche sur les sciences de la communication" CNRS-CEPEL, 1994, 60 p.

Pizzorno A., 1993 - Le radici della politica assoluta, in Le radici della politica assoluta e altri saggi, Feltrinelli, Milano.

G.Regonini - M.Giuliani : "L'Italie : au delà d'une démocratie consensuelle ?" in B.Jobert-B.Théret : Le tournant néo-libéral en Europe, L'Harmttan, Paris, 1994.

Richeri G. : "L'organisation des télécommunications en Italie" Revue Télécommunications $\mathrm{n}^{\circ} 57$, Paris, décembre 1985.

Richeri G. : "Privatisation du téléphone et intervention publique en Italie après la Seconde Guerre mondiale" in Bertho-Lavenir C. (dir.) : L'Etat et les télécommunications en France et à l'étranger 1837-1987, Librairie DROZ, Genève, 1991.

Schmitter P.C. : "Groupes d'intérêts et consolidation démocratique en Europe méridionale", Pôle Sud, 3 : 5-38. Montpellier, 1995. Smith A. : L'Europe au miroir du local. Les fonds structurels en France, en Espagne et au Royaume-Uni, L'Harmattan Logiques politiques, Paris, 1995.
N

$N \cdot O \cdot T \cdot E \cdot S$

1. A l'intérieur du groupe IRI, la holding publique STET regroupait une partie seulement des opérateurs publics : la SIP (Société Hydro-Electrique du Piémont à l'origine) gère les réseaux locaux et une partie des réseaux interurbains, Italcable est compétente pour les liaisons internationales hors Europe, Telespazio pour les communications par satellite, Italtel pour les équipements. Outre ces quatre organisations, l'ASST (Agence d'Etat pour les Services de Télécommunication), dotée du statut d'administration depuis 1925, est compétente pour une grande partie du réseau interurbain; d'autres opérateurs mineurs concentrent des activités spécifiques, comme les communications maritimes.

2. Il suffit de mentionner ici le rôle qu'ont eu les juges italiens dans la "dérégulation" du paysage audiovisuel au cours des années 1970. Cf. P.Musso, G. Pineau : L' Italie et sa télévision, Champ Vallon, 1990.

3. Le fait que Telecom Italia ait récemment été admis à faire partie de la Confédération, et qu'elle en soit aujourd'hui le plus important contributeur, n'est sans doute pas pour rien dans la difficulté de la "prise de parole" publique de la Confindustria en matière de télécommunications, de même qu'elle ne s'était exprimé que très tardivement sur la réforme institutionnelle, dans les années 1980, à cause de l'influence que détenaient 
en son sein les principaux bénéficiaires du statu quo ante (Négrier 1994)

4. Elles se sont traduites en France par la création de la Direction à la Réglementation Générale, distincte de France Télécom, en Fspagne de la DGTel, séparée de Telefonica, de même que de l'OFTEL en Grande-Bretagne

5. Un regard sur l'organigramme de Telecom Italia suffit à montrer la grande permanence des élites des différentes sociétés qui composaient la mosaïque des télécommunications italiennes. Son explication tiendrait moins, au contraire du cas français, à une logique de corps (les X-Télécom) qu'à une forme de cooptation sur la longue durée des dirigeants de ces entreprises au sein d'une "école" dont la légitimité puiserait ses racines dans ses relations avec le Vatican, certains courants démocrates-chrétiens et d'autres clubs fermés...

6. Les opérateurs français et allemand ont présenté à la Commission Européenne un projet d'alliance dans la prestation de services du secteur concurrentiel (notamment en direction des services aux entreprises). La contestation de BT (opérateur dominant et privé britannique) se fonde sur une orthodoxie libérale (l'abus de position dominante), là où l'alliance se justifie comme participant d'une politique industrielle européenne. La contradiction est forte, alors que les deux positions se raccordent à la même palette de politiques publiques européennes.

7. Et notamment la résistance à toute forme de privatisation brutale, la limitation des alliances internationales visant le territoire italien aux aspects technologiques (avec IBM), la promotion d'une autorité spécifique et distincte de l'Antitrust, la mise en œuvre, préalable à la privatisation, d'un vaste plan de câblage en fibre optique à large bande visant 10 millions de foyers à l'horizon 1999. L'annonce d'une négociation avec Cable\& Wireless sur un échange de participation, l'intégration de la STET dans le quatrième pôle international d'opérateurs C\&W-Veba-Bouygues, la privatisation annoncée et précédée d'une refonte réglementaire, la remise en question du plan de câblage au seul profit de la STET et de Telecom-Italia sont autant de revirements politiques très significatifs de l'adaptabilité de l'élite des télécoms italiennes à une contrainte environnementale qui change.

8. Cette prise de participation, qui fait le pendant à la constitution, en 1995, d'une alliance entre Olivetti et France Télécom sur le marché italien, dépasse le seul terrain des communications mobiles et vise à structurer en France, aux côtés de France Télécom et du groupe formé autour de la Générale des Eaux (avec British Telecom et l'allemand Mannesman), une concurrence sur l'ensemble du spectre des télécommunications. Cette naissance d'un opérateur généraliste français, mobilisé autour du marché de l'exploitation du réseau de télécommunication de la SNCF, s'appuie également sur la firme allemande Veba et l'affichiste Decaux.

9. cf. Il Sole 24 ore du 24 octobre 1996, pp. 6 et 14. 